\title{
Mechanical Complications after Limb Osteosynthesis: Analysis of Etiologic Factors in 42 Cases
}

\author{
Moctar Traore*, Raphael Gogoua, Maurice Kouame, Armand Yepie, Michel Anoumou, \\ Guy Varango
}

Department of Orthopaedic Surgery and Traumatology, C.H.U. Treichville, Abidjan, Cote d'Ivoire

Email: *traore_moc@yahoo.fr

How to cite this paper: Traore, M., Gogoua, R., Kouame, M., Yepie, A., Anoumou, M. and Varango, G. (2017) Mechanical Complications after Limb Osteosynthesis: Analysis of Etiologic Factors in 42 Cases. Open Journal of Orthopedics, 7, 43-52.

https://doi.org/10.4236/ojo.2017.72006

Received: November 29, 2016

Accepted: February 3, 2017

Published: February 6, 2017

Copyright $\odot 2017$ by authors and Scientific Research Publishing Inc. This work is licensed under the Creative Commons Attribution International License (CC BY 4.0).

http://creativecommons.org/licenses/by/4.0/

\begin{abstract}
The purpose of this study is to identify the causes of mechanical complications of osteosynthesis of members to prevent and improve the results of our surgical treatments. Patients and Method: Retrospective study including all patients diagnosed with mechanical complications after osteosynthesis of the limbs and who received medical follow-up. 42 patients with an average age of 42.2 years, with a male predominance ( 36 cases) were selected. These complications predominated in the femur with 26 cases. The implants involved were predominantly screwed plates with 25 cases. We investigated and analyzed the different types of dismantling of material as well as the etiological factors that may be at the origin of these disassembly. Results: After a mean follow-up of 15.47 months, four types of complications were identified: material expulsion (20 cases), migration ( 3 cases), incurvation ( 7 cases), fracture of material (12 cases). 4 types of factors causing dismantling have been identified: the factors related to the patient, factors related to fracture, the factors related to the implant and factors related to the surgeon. Conclusion: Disassembly of orthopedic implants is most often of multifactorial origin. These factors may act in isolation but are most often entangled in the patient. Proper preoperative planning can help prevent them. However, unexpected stresses of the implant and certain factors related to the ground remain difficult to control.
\end{abstract}

\section{Keywords}

Pin, Nail, Plate, Screws, Mechanical Complications, Osteosynthesis

\section{Introduction}

The mechanical complication of an osteosynthesis is defined as permanent deformation or rupture of the osteosynthesis material or its support [1]. Osteo- 
synthesis places the bone segment in new mechanical conditions that evolve throughout the periods of consolidation and mobilization. In addition to these, mechanical complications of various kinds can be observed. Several factors are responsible for these relatively frequent mechanical complications. This frequency varies between $6 \%$ and $18 \%$ according to the authors [1] [2] [3]. This retrospective study allowed us to identify and analyze the most frequent causes of these complications in order to prevent them.

\section{Patients and Method}

This is a retrospective study for analytical purposes over 4 years (January 2012 to December 2015). All patients operated for limb osteosynthesis using various implants and whose mechanical failure of the osteosynthesis was diagnosed and treated in our service were included in this study. We excluded from the study all patients who presented a mechanical complication after bone consolidation as well as patients operated for arthroplasty. During the study period, $1514 \mathrm{limb}$ osteosynthesis were performed, including 480 in the upper limb and 1034 in the lower limb. The implants used were distributed as follows: screwed plates 810 , centromedullary nails 320 , external fixation 305 , simple screwing 27 , broaching 52. After the hospital discharge, all these patients underwent clinical and radiological follow-up on a monthly basis. External consultation was until bone consolidation and during functional rehabilitation sessions. During this postoperative follow-up, 42 mechanical complications were diagnosed $(2.77 \%$ of limb osteosyntheses). All these patients were followed and treated in the service. The mean age was 42.2 years with extremes from 18 years to 86 years. There was a male predominance with 36 cases, $(85.71 \%)$. Dismantling predominated in the femur with more than half the cases (26 cases, 62\%), including 18 diaphyseal, 5 proximal epiphyseal and 2 distal epiphyseal); 10 cases in the leg, 3 cases in the humerus, 2 cases in the forearm and 1 case in the clavicle. Table 1 shows the distribution of complications according to the site of osteosynthesis. The affected implants were mostly plates with 25 cases; 4 single screwings, 5 spindles and 7 centromedullary nailings were involved. These implants all came from the same supplier of synthetic material and the same manufacturer. We investigated and analyzed the different types of dismantling of material as well as the etiological factors that may be at the origin of these disassembly.

Table 1. Socio-demographic and clinical characteristics of patients.

\begin{tabular}{|c|c|c|c|c|c|c|c|c|c|c|}
\hline Age moy & \multicolumn{2}{|c|}{ Sexe } & \multicolumn{2}{|c|}{ Profession } & \multicolumn{2}{|c|}{ Siege de la fracture } & \multicolumn{2}{|c|}{ Matériel } & \multicolumn{2}{|c|}{ Complications } \\
\hline \multirow{5}{*}{42.2 ans } & & & Student & $7(16.6 \%)$ & Clavicle & $01(2.3 \%)$ & Plate & $25(59.5 \%)$ & Incurvaison & $07(16.6 \%)$ \\
\hline & & & Worker & $24(57.1 \%)$ & Humérus & $03(7.1 \%)$ & Screw & $04(9.5 \%)$ & Expulsion & $20(47.6 \%)$ \\
\hline & M & $\mathrm{F}$ & Military & $7(16.6 \%)$ & Forarm & $02(4.7 \%)$ & Brooch & $05(11.9 \%)$ & Migration & $03(7.1 \%)$ \\
\hline & & & Unemployed & $4(09.5 \%)$ & Fémur & $26(61.9 \%)$ & Nail & $08(19.0 \%)$ & Rupture & $12(28.5 \%)$ \\
\hline & & & & & Leg & $10(23.8 \%)$ & & & & \\
\hline Total & \multicolumn{2}{|c|}{42} & \multicolumn{2}{|c|}{42} & \multicolumn{2}{|c|}{42} & \multicolumn{2}{|c|}{42} & \multicolumn{2}{|c|}{42} \\
\hline
\end{tabular}




\section{Results}

After a mean follow-up of 15.47 months, four types of méchanical complications were identified and treated: material migration (3 cases), materiel expulsion (20 cases), incurvation of the materiel (7 cases), fracture or rupture of material 12 cases). Table 1 shows the socio-demographic and clinical characteristics of the patients.

The migration of the material (Figure 1) is defined as the progresson or the depression of the material beyond the supposed normal limits. It was observed with a screw after osteosynthesis of femoral neck fracture. We also observed the migration of two tibial nails to the loading of the patient 3 months after osteosynthesis. A surgical revision was indicated for non-consolidation: ablation of the tibial nail, osteosynthesis by targeted plate associated with a bone graft.

The expulsion of the material we have defined as the receding or loosening of the material has been observed with screws (involved screws) (loosening of the screw) (Figure 2). In the majority of cases, the screws were made of a femoral screw plate (10 cases), a humeral plate, 4 tibial plates and a front arm plate. The expulsion also involved 2 insulated screw connections-fracture of the femoral condyles, 2 screwings of non-displaced fractures of the neck of the femur.

The incurvaison or unruptured permanent deformation of the material (Figure 3) was observed only in the lower limb and involved the plaques (3 cases including 2 on the femur and 1 on the tibia) and nails (6 cases all on the femur). A reoperation was necessary for lack of axis and or shortening of the limb. Bone graft was not required.

As for the fracture or rupture of the material (Figure 4), it was also observed exclusively in the lower limb (12 cases) and in 8 cases of rupture of the screws and in 4 cases of fracture of the femoral screw plate. The majority of these patients (10 out of 12 ) had been treated early prior to bone consolidation. One

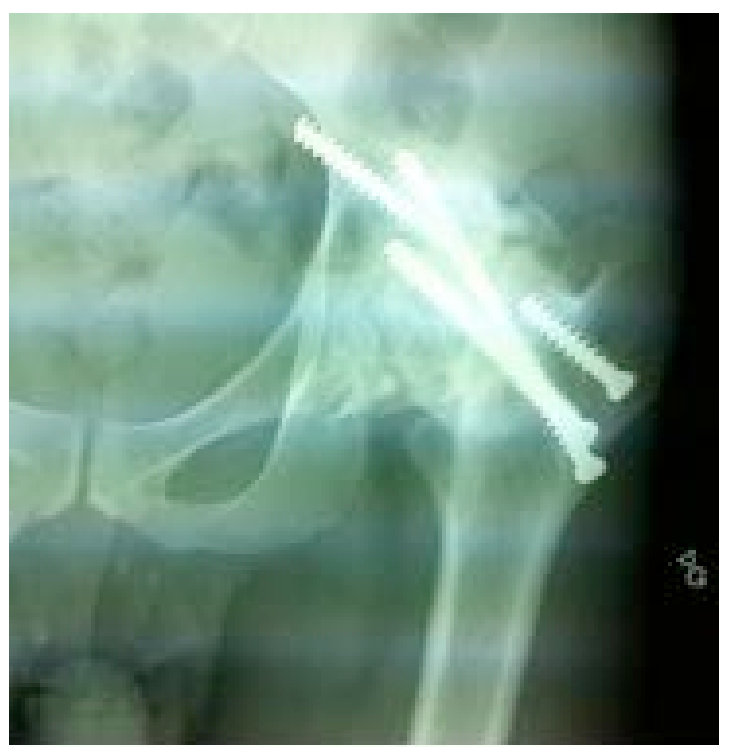

Figure 1. Fracture and migration of screw in pelvic after ostéosynthesys of femoral neck fracture. 


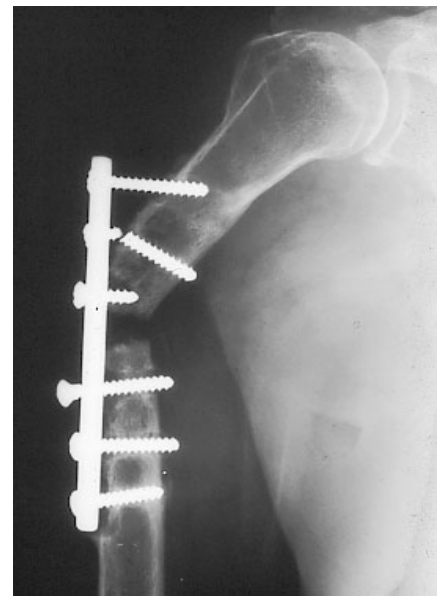

Figure 2. Expulsion of screws after osteosynthesys of humeral fracture.

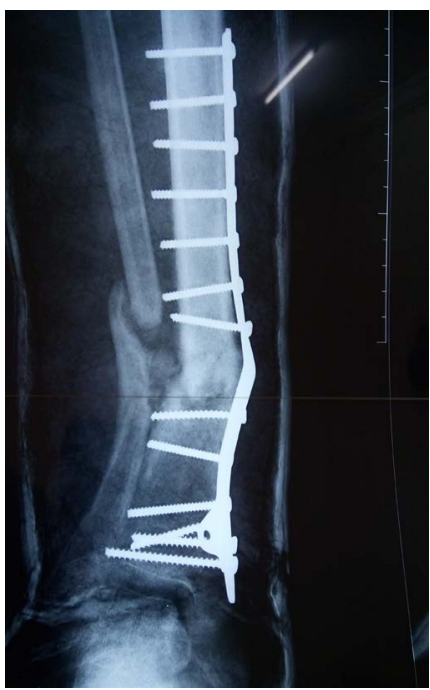

Figure 3. Incurvaison of plate after osteosynthesys of tibial fracture.

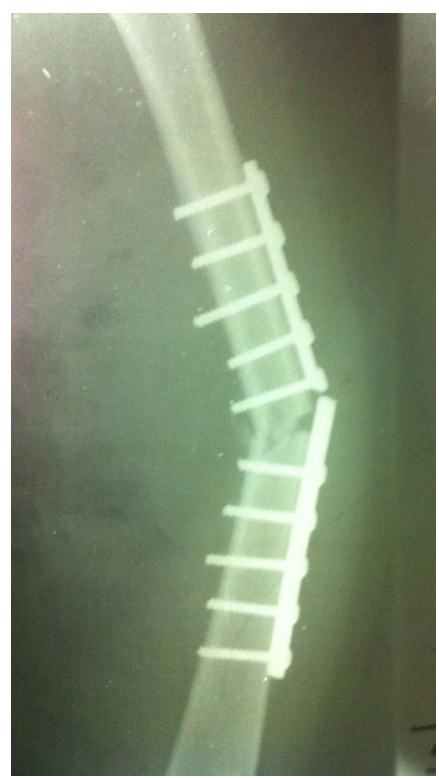

Figure 4. Rupture of plate after femoral diaphyseal osteosynthesys. 
patient took support after leaving the hospital on day 5 after a femoral plate osteosynthesis. The reoperation for absence of bone consolidation was carried out for 6 patients with bone graft in 4 cases. The 6 others had their material simply removed because they had already consolidated. Table 2 shows the distribution of mechanical complications according to the following up.

The causes of these mechanical complications were investigated and grouped into 4 types of etiologic factors: patient-related factors (age, indiscipline, weight, infection); factors related to the fracture: the seat and the anatomo-radiological type of the fracture (comminution, the existence of several fragments); the factors related to the implant (the bad choice of the implant, the maladjustment); factors related to the surgeon: the surgical technique, the technical errors.

- Patient-related factors:

The etiologic factors most commonly encountered in our study were those related to the patient. Failure to follow postoperative medical instructions with mobilization and early loading was the most common cause in the lower limb (26 cases). 5 of our patients were osteoporotic (aged over 70 years). Only a teenager of 15 years. The bone fragility of the child and the elderly person would explain some expulsions of screws; whereas the bone strength observed in young adults could be the cause of fractures or ruptures of material. An infection of the surgical site was noted in $3(7.42 \%)$ of our patients after osteosynthesis by femoral plate. This was the case in 2 cases of screw expulsion and in the case of a plate rupture. The diagnosis of the infection has been evoked after the discovery of the dismantling of the material and confirmed in intraoperative during the surgical resumption.

- Factors related to fracture:

The complexity of the fracture including the existence of a comminution was observed in $70 \%$ of the cases and could be the cause of dismantling of material.

- Factors associated with the implant:

Concerning the implants, all the nails for which a mechanical complication was observed were unlocked nails (kuncher nails). In addition, the screws we use are non-self-locking screws. These factors would favor expulsions and incurvations of the implant

- Factors related to the surgeon:

The surgeon and especially the technical errors were at the origin of some mechanical complications; In fact, we observed technical defects on some radiographs of post-operative control: it was most often,

-an internal defect on the opposite side to the screwed plate or a reduction defect with the persistence of a diastasis was found in 8 cases,

Table 2. Distribution of mechanical complications according to the following up.

\begin{tabular}{cccccc}
\hline & Migration & Expulsion & Incurvaison & Rupture & Total \\
\hline $\mathbf{n}(\%)$ & $03(7.1 \%)$ & $20(47.6 \%)$ & $07(16.6 \%)$ & $12(28.5 \%)$ & 42 \\
Follow up (months) & 10.5 & 16.6 & 15.1 & 18.6 & 15.4 \\
\hline
\end{tabular}


-the presence of one or more non-screwed holes or intrafocal screws: 6 times, -of a plate of insufficient length, width or thickness: 2 times,

-a centromedullary nail of inadequate length (2 times). These defects were isolated or associated in the same patient.

\section{Discussion}

Mechanical complications after limb osteosynthesis ranged from $6 \%$ to $18 \%$ according to the literature (Benoit [2] found 18\%, Riemer [4] 7\%, and $11 \%$ for Wagner [5]. We have grouped into 4 types.

- Patient-related factors:

Age: in young subjects $<15$ years of age, cortical and thin, canals of havers occupy the major part of the bone, giving it an areolar aspect [6] [7]. This makes the holding of the screws more random and favors their disassembly of the screws by tearing (expulsion). Essadki et al. [8] found 50\% mechanical complications in subjects younger than 15 years. This was difficult for us because our service is exclusively reserved for the osteo-articular surgery of the adult. In the adult the cortical bone is very solid favoring better inking. Disassembly in adults is therefore more often due to breakage of screws or plaque than to expulsion. This was confirmed by our study because the 12 cases of rupture or fracture of material that was observed in adults. In the elderly $>60$ years, there is an osteoporosis with a thinning of the cortical which is the factor often at the origin of expulsion of the screws. Osteoporosis is found in almost all authors. (Our series: 5 patients $>60$ years including 2 very osteoporotic)

The weight of the patient: overweight or obesity is the factor of mechanical stress at the origin of rupture or expulsion of material.

The indiscipline of the patient at the origin of the mobilizations and the early loadings without medical advice are common factors incriminated. Early loading was found in all patients (100\%) in the series of Moyikoua et al. [9], which involved only mechanical complications in the lower limb. Ibrahimou et al. [10] found $50 \%$ early loading as a cause of femoral nail curvature. In the Essadki study [8], early loading accounted for $54 \%$ as a cause of incurvation and screwed plate fracture, with our 26 patient series accounting for $61 \%$.

Infection, when it acts by causing locally a bone lysis around the material, reduces the quality of the inking. It was found in only $3(7.14 \%)$ of our patients. Its role in the occurrence of mechanical complications of osteosynthesis has not been frequently demonstrated in the literature because the majority of studies have dealt with aseptic mechanical complications. Gogoua et al. [11], however, found a higher rate (38\%) of local infection in their series.

- Factors related to the fracture:

The seat and the type of the fracture are sometimes incriminated in the occurrence of mechanical complications. Indeed in the complex comminutives or multi-fragmentary fractures it is the material which undergoes all the constraints due to the absence of inter-fragmentary contact. Attempts to reduce the anatomy of the fragments may be to the detriment of their vascularization, com- 
promising the consolidation. Some authors (Ruedi and luscher [12]) have reported the possibility of consolidation of complex fractures by simple bridging equipment without seeking the anatomical reduction advocated by several schools. Moyikoua et al. [9] had 12 patients out of 22 (54\%) who had complex fractures. For Burny et al. [1], fracture was the only cause of disruption in 70 patients. We found it in 29 patients (70\%). At the site of the fracture, the cortical thickness determines the quality of fixation of the screws. Indeed, based on the morphological subdivision of the diaphysis of the long bones, especially of the femur, advocated by Benoit et al. [2], the cortex is thicker in 1/3 medium allowing a good anchoring of the screws, while the $1 / 3$ proximal and distal have a less thick cortex with thus less anchoring of the screws thus increasing the risk of dismantling by tearing. At the site of the fracture, the cortical thickness determines the quality of fixation of the screws. Indeed, based on the morphological subdivision of the diaphysis of the long bones, especially of the femur, advocated by Benoit et al. [2], the cortex is thicker in $1 / 3$ medium allowing a good anchoring of the screws, while the $1 / 3$ proximal and distal have a less thick cortex with thus less anchoring of the screws thus increasing the risk of dismantling by tearing.

- Factors related to the implant:

The implant must be adapted to the fracture. According to Meyrueis J [13], for a better stability of the osteosynthesis, the plates must be sufficiently long, thick and wide allowing a sufficient number of screws on both sides of the fracture focus and good rigidity. According to him, the stiffness depends on its dimensions represented by the formula: $\mathrm{I}=\mathrm{L} . \mathrm{e} 3 / 12(\mathrm{I}=$ moment of inertia in flexion, $\mathrm{L}=$ width, $\mathrm{e}=$ thickness).

Closed focus osteosynthesis should be preferred to the open hearth, which increases the risk of infection and therefore dismantling of the synthetic material. The use of anti-rotatory and dynamic systems increases the stability of the osteosynthesis preventing the expulsion, rotation or migration of the implants. Several studies have thus shown a better stability of the osteosyntheses of the fractures of the proximal femur with the use of the antirotatory systems [14] [15].

The use of anti-rotatory and dynamic systems increases the stability of the osteosynthesis preventing the expulsion, rotation or migration of the implants. Several studies have thus shown a better stability of the osteosynthesis of the fractures of the proximal femur with the use of the antirotatory systems [14] [15].

The locked nailing of Kemfp and Gross [16] has indeed improved the prognosis of complex and comminuted fractures. The stability of the rotational and axial mounting is improved by the locking. The centromedullary nail whose migration we observed was unlocked. The same observation was made by Moyikoua [9] in Togo and Wami [17] in the DRC. We do not use self-locking plates or screws that considerably reduce the risk of disassembling the equipment [18].

- Factors related to surgery

The implantation of an implant can be the source of several technical errors 
whose role in the genesis of mechanical complications is certain but difficult to meter prominently posteriori. These technical errors are reported by all authors with variable rates: Essadki [8] described 2 cases out of 31, Gogoua [11] 13 out of 26, Arnaout [19] gamma nail. Eight of our patients (19\%) had an internal bone defect (opposite side of the plate) and/or persisted diastasis on postoperative radiographs; This could be the reason for the dismantling of the equipment. Indeed Muller [20] has demonstrated that the existence of an internal defect or an inter-fragmentary diastasis subjects the material to flexion-compression and flexion-traction stresses leading to the fatigue of the material causing its curvature Which may go as far as the rupture of the interest of the filling of the defects by a graft (Müller). In 6 of our patients (14\%), there were non-target plate holes or screws in the fracture line. According to Muller [20] a minimum of 4 screws that is to say 7 to 8 cortical are indispensable for a better osteosynthesis by screwed plate. The presence of an intrafocal screw can lead to a yawning of the fracture focus with poor inter-fragmentary contact subjecting the material to stresses. The length and the unsuitable diameter of the centromedullary nails were responsible for 2 nail migrations in our patients. This complication has been observed in several authors [21] [22] [23].

The complexity of the fracture ( $70 \%$ of the cases) associated with the indiscipline of the patients ( $61 \%$ of the cases) are the factors most commonly found in the genesis of these mechanical complications.

\section{Conclusion}

Mechanical complications after limb osteosynthesis are most often of multifactorial origin. These factors may act in isolation but are most often entangled in the patient. Proper preoperative planning can help prevent them. However, unexpected stresses of the implant and some factors related to the ground remain difficult to control.

\section{Limits of the Study}

This study is limited by its retrospective nature, the small sample size and the impossibility of achieving other.

Mechanical complications after limb osteosynthesis. A case-control study on a larger sample will be necessary for future study.

\section{Conflict of Interests}

The authors declare no conflict of interest.

\section{Author's Contributions}

This study was approved by the local ethics committee and all the authors contributed to the writing of this article.

\section{References}

[1] Burny, F., Bourgois, R. and Lemaire, L. (1974) Failure of the Osteosynthesis 
Material. Implication of the Implant. Acta Orthopædica Belgica, 37, 846-860.

[2] Benoit, J., Corotteau, Y. and Tomeno, B. (1974) Critical Study of Failures in the Treatment of Fresh Fractures of the Femoral Diaphysis: About 330 Cases. Revue De Chirurgie Orthopedique et Traumatologique, 60, 465-482.

[3] Pidhorz, L., Raguin, J. and Varenne, F. (1975) The Osteosynthesis Materials. Encycl. Med Chir, Paris, Surgical Techniques, 1-10.

[4] Riemer, B.L., Butterfiel, S.L., Burke, C.J. and Matthews, D. (1992) Immediate Plate Fixation of Comminuted Femoral Diaphyseal Fractures Hydhly in Blunt Polytrauma Patients. Orthopedics, 15, 907-915.

[5] Wagner, R. and Weckbach, A. (1994) Komplikationen plattenosteosynthese am femurchaft. Eine analysis from 199 femurfrakturen. Unfallchirurg, 97, 139-143.

[6] Currey, J.D. and Butler, G. (1975) The Mechanical Properties of Bone Tissue in Children. The Journal of Bone and Joint Surgery, 57-A, 810-814.

https://doi.org/10.2106/00004623-197557060-00015

[7] Hirsh, C. and Evank, F.G. (1965) Some Studies of Physical Properties of Compact Bone Infante. Acta Orthopaedica Scandinavica, 35, 300-305. https://doi.org/10.3109/17453676508989361

[8] Essadki, B. and Lamine, A. (2000) The Aseptic Mechanical Complications of Fractures of the Femoral Diaphysis Treated by Screwed Plates. Acta Orthopædica Belgica, 66, 61-68.

[9] Moyikoua, A., Bouty-Buang, J.C. and Pena Pitra, B. (1993) Post-Operative Mechanical Complications of Osteosyntheses of the Lower Limb. Analysis of 22 Cases. Medenine Afrique Noire, 40, 509-515.

[10] Ibrahimou, T.K., Ibrahimou, B., Makekita, W.N. and Mudimisi, M. (2016) The Mechanical Complications of the Centromedular Nailing of the Femur in a Precarious Environment. About 6 Observations at the N'djili Hospital, Kinshasa. Revue Médicale des Grand Lacs, 5, 96-103.

[11] Gogoua, R.D., Toure, S., Anoumou, M., Kouame, M., Kone, B. and Varango, G.G. (2006) Mechanical Complications of Osteosynthesis of Limb Fractures: An Epidemiological Analysis of 26 Observations. Mali Medicale, 21, 5-8.

[12] Ruedi, T.P. and Lusher, J.N. (1979) Result after Internal Fixation of Comminuted Fractures of the Femoral Shaft with DC Plates. Clinical Orthopaedics, 138, 74-76.

[13] Meirueis, J.P., Meirueis, J. and Sohier, M.A. (1975) Materials Used for Osteosynthesis. Encycl Med Chir (Paris-France) Surgical Techniques Orthopedics Traumatology, 44-015A.

[14] De Landevoisin, E.S., Bertani, A., Candoni, P., Charpail, C. and Demortiere, E. (2012) Proximal Femoral Nail Antirotation (PFN-ATM) Fixation of Extra-Capsular Proximal Femoral Fractures in the Elderly: Retrospective Study in 102 Patients. Orthopedics and Traumatology. Surgery and Research, 98, 288-295. https://doi.org/10.1016/j.otsr.2011.11.006

[15] Loubignac, F. and Chaba, J.F. (2009) A Newly Designed Locked Intramedullary Nail for Trochanteric Hip Fractures Fixation: Results of the First 100 Trochanteric Implantations. Orthopaedics and Traumatology Surgery and Research, 95, 139-144. https://doi.org/10.1016/j.otsr.2009.01.002

[16] Kempf, L., Big, A. and Lafforgue, D. (1978) Fixing a Fracture Should It Be Rigid or Elastic? Revue Chirurgie Orthopedique et Traumatologique, 64.

[17] W'ifongo, W., Lembalemba, B., Kalokola, L. and Afelokoky, E. (2014) Migration and Incurvation of the Nail, Two Complications of the Centromedilar Nailing of the Femur on a Case Observed at the University Clinics of Kisangani, DRC. Kisangani 
Medical, No. 2, 104-108.

[18] Roll, D.M., Laflamme, G.Y., Berry, G.K., Harvey, E.J., Delisle, J. and Girard, J. (2009) Self-Locking Plate Osteosynthesis Percutaneous Fractures of the Proximal Humerus. Revue Chirurgie Orthopedique Traumatologique, 95, 57-63.

[19] Arnaout, A., Beya, B., Delplace, J., Vial, D. and Lecestre, P. (1993) Osteosynthesis of Fractures of the Trochanteric Region by Gamma Nail: About 76 Cases Reviewed. Acta Orthopaedica Belgica, 59, 31-39.

[20] Muller, M.E., Algower, M., Sneider, R. and Willeneger, H. (1980) Manuel Osteosynthesis. AO Technique, Springer Verlag, Berlin.

[21] Decoster, T.A. and Miller, R.A. (2003) Closed Intramedullary Nailing Locked of Femoral Shaft Fracture in Elderly. The Iowa Orthopaedic Journal, 23, 43-45.

[22] Tesson, A. (2004) The Complications of the Centromedullary Nailings of the Long Bones Bearing about 397 Cases. Thesis of Doctorate, No. 124, University of Nante, Nante.

[23] Hunter, S.G. (1982) The Migration of the Femoral Nail Kuntcher. A Clinical Study. Injury, 13, 460-463. https://doi.org/10.1016/0020-1383(82)90159-0

Submit or recommend next manuscript to SCIRP and we will provide best service for you:

Accepting pre-submission inquiries through Email, Facebook, LinkedIn, Twitter, etc. A wide selection of journals (inclusive of 9 subjects, more than 200 journals) Providing 24-hour high-quality service User-friendly online submission system Fair and swift peer-review system Efficient typesetting and proofreading procedure Display of the result of downloads and visits, as well as the number of cited articles Maximum dissemination of your research work

Submit your manuscript at: http://papersubmission.scirp.org/

Or contact ojo@scirp.org 